\title{
Load Modulation of BOLD Response and Connectivity Predicts Working Memory Performance in Younger and Older Adults
}

\author{
Irene E. Nagel ${ }^{1,2}$, Claudia Preuschhof ${ }^{1,2}$, Shu-Chen $\mathbf{L i}^{1}$, Lars Nyberg ${ }^{3}$, \\ Lars Bäckman ${ }^{1,4}$, Ulman Lindenberger ${ }^{1}$, and Hauke R. Heekeren ${ }^{1,2}$
}

\begin{abstract}
Individual differences in working memory (WM) performance have rarely been related to individual differences in the functional responsivity of the WM brain network. By neglecting person-to-person variation, comparisons of network activity between younger and older adults using functional imaging techniques often confound differences in activity with age trends in WM performance. Using functional magnetic resonance imaging, we investigated the relations among WM performance, neural activity in the WM network, and adult age using a parametric letter $n$-back task in 30 younger adults (2131 years) and 30 older adults (60-71 years). Individual differ-
\end{abstract}

\section{INTRODUCTION}

The function of working memory (WM) is to maintain and manipulate information on-line (Jonides et al., 2008; D'Esposito, 2007; Baddeley, 2003). Individual differences in WM ability are large (Schmiedek, Lövdén, \& Lindenberger, 2009; Miyake, 2001) and increase with age (de Frias, Lovden, Lindenberger, \& Nilsson, 2007). How such heterogeneity in performance relates to differences in functional brain activation patterns is not well understood. Individuals might differ in the amplitude of local brain activation and, crucially, also in the connectivity of regions within the WM network.

The brain substrates of WM consist of a distributed fronto-posterior network including lateral prefrontal cortex (PFC, middle and inferior frontal gyrus), the supplementary motor area (SMA), premotor cortex (PMC), and posterior parietal cortex (PPC; D'Esposito, 2007; Owen, McMillan, Laird, \& Bullmore, 2005; Miller \& Cohen, 2001; Jonides et al., 1997; Jonides et al., 1993). Anatomically, the regions of the WM network are densely interconnected (Barbas \& Zikopoulos, 2007; Croxson et al., 2005; Mesulam, 1990; Barbas \& Pandya, 1989; Petrides \& Pandya, 1984). Furthermore, strong task-related coupling among regions of the WM network, including fronto-polar cortex (FPC), dorsolat-

\footnotetext{
${ }^{1}$ Max Planck Institute for Human Development, Berlin, Germany,

${ }^{2}$ Freie Universität Berlin, Germany, ${ }^{3}$ Umeå University, Sweden,

${ }^{4}$ Karolinska Institute, Stockholm, Sweden
}

ences in the WM network's responsivity to increasing task difficulty were related to WM performance, with a more responsive BOLD signal predicting greater WM proficiency. Furthermore, individuals with higher WM performance showed greater change in connectivity between left dorsolateral prefrontal cortex and left premotor cortex across load. We conclude that a more responsive WM network contributes to higher WM performance, regardless of adult age. Our results support the notion that individual differences in WM performance are important to consider when studying the WM network, particularly in age-comparative studies.

eral PFC (DLPFC), ventrolateral PFC (VLPFC), SMA, anterior cingulate cortex (ACC), thalamus, PPC, and visual cortex, has been reported in functional magnetic resonance imaging (fMRI) connectivity studies (Chang, Crottaz-Herbette, \& Menon, 2007; Postle, 2006; Kondo, Morishita, et al., 2004; Kondo, Osaka, \& Osaka, 2004; Glabus et al., 2003; Honey et al., 2002; Della-Maggiore et al., 2000).

The $n$-back task is often used to assess WM (e.g., Owen et al., 2005). In the verbal version of the $n$-back task, participants watch a sequence of letters on the screen and indicate for each letter by a button press whether the letter they currently see is the same as the one they had seen previously. Task difficulty can be varied by instructing the participant to compare the current letter to the letter 1-back, 2-back, or 3-back (cf. Figure 1A).

In young adults, the brain responds to higher levels of WM load with increased blood oxygen level dependent (BOLD) signal in many regions of the WM network (Nyberg, Dahlin, Stigsdotter Neely, \& Bäckman, 2008; Callicott et al., 1999; Rypma, Prabhakaran, Desmond, Glover, \& Gabrieli, 1999; Barch et al., 1997; Braver et al., 1997; Jonides et al., 1997; Klingberg, O'Sullivan, \& Roland, 1997; Manoach et al., 1997). The BOLD response typically increases linearly up to a certain level of load and then levels off or declines. The change in activation with load can thus be described with a nonlinear dose-response function relating BOLD response to WM load, often following the shape of an inverted $\mathrm{U}$. It has been suggested that the point of maximum 
Figure 1. (A) Experimental task design of letter $n$-back task A series of letters is presented. The task is to indicate by a button press for each letter whether it is the same as the one presented 1-back, 2-back, or 3-back depending on the condition. (B and $\mathrm{C}$ ) Behavioral performance during the letter $n$-back task. (B) Accuracy and (C) response times (RT) for young and older adults at the three different load levels. Significant effects of Age, Load, Age $\times$ Load for both accuracy and correct RT at $p<.005$ (trend level for Age $\times$ Load interaction for accuracy).

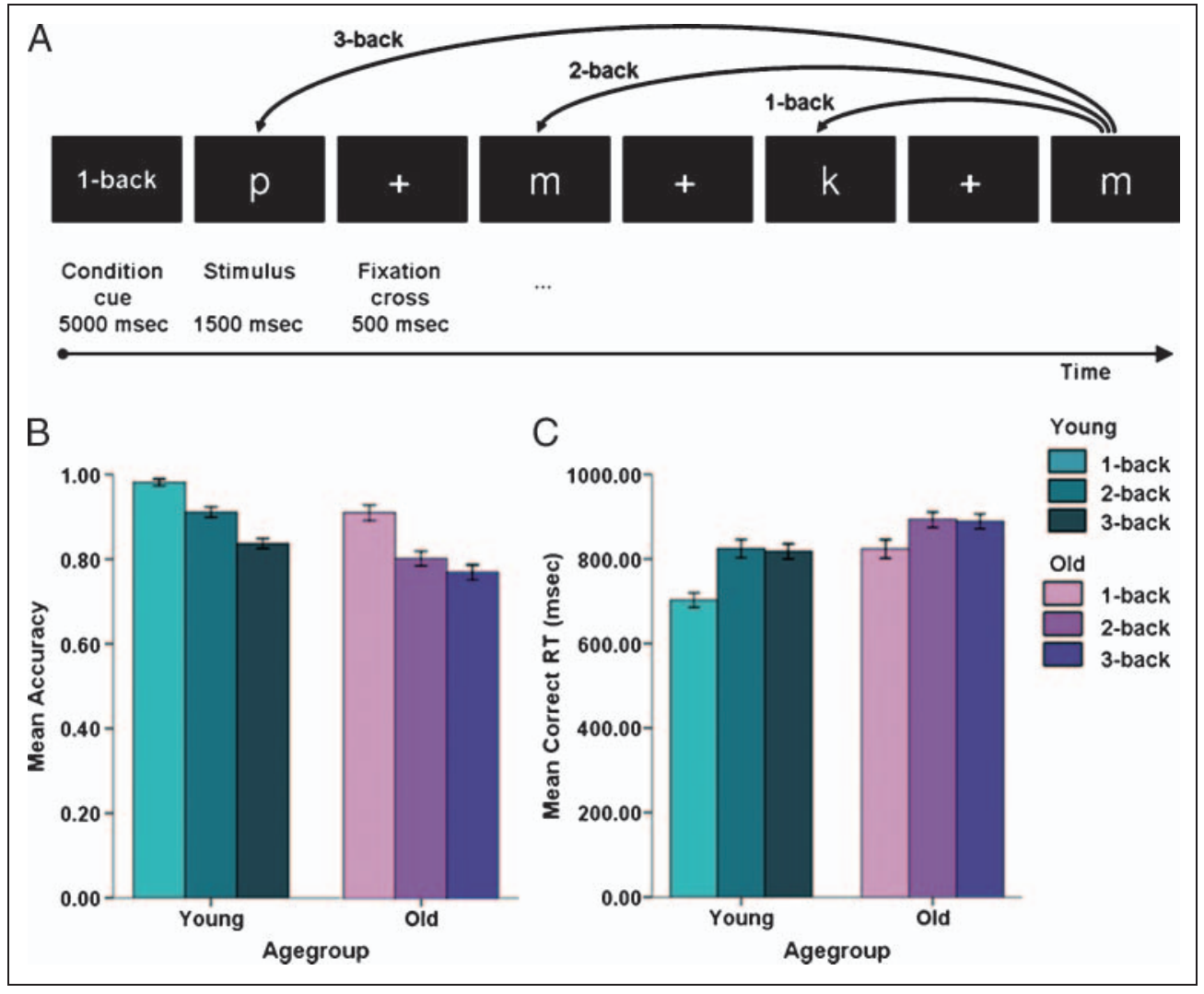

activation is a neural indicator of the WM capacity limit (Todd \& Marois, 2005; Callicott et al., 1999). Behavioral evidence demonstrates that individual differences in WM performance are large throughout adulthood (Conway, Jarrold, Kane, Miyake, \& Towse, 2008; Mayr \& Kliegl, 1993). Levels of activation in regions of the WM network have been related to WM performance (Todd \& Marois, 2005), but the precise relations between load-related changes in brain activation and performance are not yet known.

WM deteriorates markedly with advancing adult age (Babcock \& Salthouse, 1990). The brain undergoes a variety of changes that affect the integrity of gray and white matter (Raz, 2005). These changes are particularly pronounced in brain regions supporting WM functioning, such as PFC and PPC (Raz et al., 2005; West, 1996). Furthermore, WM-relevant neurotransmitter systems, particularly the dopamine system (Bäckman, Nyberg, Lindenberger, Li, \& Farde, 2006), decline with age. Relative to young adults, functional imaging studies often report overactivation at low task load in older adults (e.g., Reuter-Lorenz \& Lustig, 2005). As task difficulty increases, however, older adults tend to show a more constrained BOLD response than younger adults, particularly at high load (Nyberg et al., 2008; Mattay et al., 2006). These findings are in accordance with the Compensation-Related Utilization of Neural Circuits Hypothesis (CRUNCH), according to which older adults show overactivation at low load, presumably as a compensatory response to neurobiological decline. At higher load, activation cannot be further increased, leading to a compro- mised BOLD response (Cappell, Gmiendl, \& Reuter-Lorenz, 2010; Reuter-Lorenz \& Cappell, 2008). Schneider-Garces and colleagues (2010) showed that load-related changes in BOLD activation are linked to load-dependent changes in memory span indicating the importance of neural responsivity for task performance. Except for this study and a study by Nagel et al. (2009), differences between age groups in BOLD responsivity to increasing WM task demand were typically examined by averaging across individuals within age groups, so that individual differences in WM performance and BOLD response within age groups could not be examined. High- versus low-performing subgroups of younger and older adults have been examined in a number of papers including those of Duverne, Motamedinia, and Rugg (2009), Gazzaley, Cooney, Rissman, and D'Esposito (2005), as well as Cabeza, Anderson, Locantore, and McIntosh (2002). These studies, however, focused mainly on episodic memory and did not examine individual differences in BOLD responsivity to increasing task difficulty.

Connectivity studies show that the modulation of WM load affects not only local activation patterns but also the connectivity among regions of the WM network. Using a verbal $n$-back task with different memory loads, Honey et al. (2002) found in young adults that the coupling among regions within $\mathrm{PFC}$ and between $\mathrm{PFC}$ and parietal cortex increased with load. Similarly, Narayanan et al. (2005) reported that connectivity between DLPFC and PPC increases with load, and Axmacher, Schmitz, Wagner, Elger, and Fell (2008) reported that connectivity between 
medial temporal lobe (MTL) and visual regions increases and becomes more specific with increasing load in a visual WM task. Also, research not involving a load manipulation reveals a link between connectivity patterns and task performance (e.g., Kirchhoff \& Buckner, 2006).

Age-related differences in connectivity patterns have been associated with age-related reductions in white matter integrity (O'Sullivan et al., 2001; Gunning-Dixon \& Raz, 2000; Pfefferbaum et al., 2000; Cabeza, McIntosh, Tulving, Nyberg, \& Grady, 1997). fMRI studies comparing functional or effective connectivity of younger and older adults typically show that connectivity decreases with age (Damoiseaux et al., 2008; Sambataro et al., 2008; Andrews-Hanna et al., 2007; Cook, Bookheimer, Mickes, Leuchter, \& Kumar, 2007; Bennett, Sekuler, McIntosh, \& Della-Maggiore, 2001). Such declines in connectivity have been related to decreased performance in cognitive tasks (e.g., Andrews-Hanna et al. 2007). Importantly, though, none of these previous studies directly investigated the relation between individual differences in load-related changes in connectivity and WM performance. Thus, it remains unclear how the fine-tuning of connections within the WM network as a response to increased task demand is related to performance across the lifespan.

Thus, in general, prior research shows that processing within the WM network is modulated by load, changes with age, and is related to performance levels. It is still unclear, however, how individual differences in WM performance are related to processing within the WM network. Findings from aging research suggest that, in older adults, adjustment of brain activations to task demand (responsivitiy) is compromised (Cappell et al., 2010; Schneider-Garces et al., 2010; Park \& Reuter-Lorenz, 2009; Reuter-Lorenz \& Lustig, 2005; Reuter-Lorenz, 2002; Grady et al., 1998). Here we investigate how individual differences in responsivity are related to performance. We propose to extend the concept of responsivity to the adult population in general by suggesting that greater BOLD responsivity should be related to better task performance at any adult age. Furthermore, based on findings related to connectivity and WM performance, we propose that not only changes in local processing but also the adjustment of coordinated processing of connected brain regions as a response to increasing task demand is relevant for proficient performance.

We examined whether and how responsivity to WM challenge of both local functional brain activation and connectivity between WM regions is related to task performance in younger and older adults. To investigate responsivity, we administered a letter $n$-back task with three load levels (Figure 1A) to 30 younger and 30 older adults using fMRI. First, we hypothesized that age groups would differ in their responsivity with older adults having a compromised response compared to younger adults. Second, we expected that responsivity of the local BOLD response would explain variation in performance beyond age group membership. Finally, we predicted that individual differences in responsivity of distributed processing (thus, in ef- fective connectivity within the WM network) would be related to individual differences in WM performance.

\section{METHODS}

\section{Participants}

Thirty younger and 30 older adults participated in the study (see Table 1 for demographic details). We recruited participants via newspaper announcements and from a database of the Max Planck Institute for Human Development in Berlin. All participants were right-handed, had normal or corrected-to-normal vision, no history of neurological or psychiatric disease, and did not take psychiatric medication. For the older adults, we acquired additional information about their mental status (Mini Mental State Exam), white matter hyperintensities (WMH), and vascular risk factors. The mean Mini Mental score was above 26 indicating that the older sample was cognitively healthy. WMH volume was at a mean of 1502.14 voxels $(S D=$ 1436.37). Vascular risk factors (data missing for 5 participants): 12 participants had arterial hypertension (defined as blood pressure constantly above 140/90 mm Hg), 7 of the 12 hypertensive participants took antihypertensive medication. Four participants had type 2 diabetes, all of whom took medication, but only one was insulin dependent. Three participants reported hypercholesterolemia. The ethics committee of the Charité University Medicine, Berlin, approved the study. Written informed consent was obtained from participants prior to investigation. Participants received financial reimbursement.

\section{Neuropsychological Tests for Sample Description}

Participants completed two additional cognitive tasks: the Digit Symbol Test, which is a test of fluid intelligence, and the Spot-a-Word Test, which is a test of crystallized intelligence. The Digit Symbol Test is a subtest of the Wechsler Adult Intelligence Scale-Revised (Wechsler, 1981). The Spot-a-Word Test (Lindenberger, Mayr, \& Kliegl, 1993) is a forced-choice test that requires participants to spot real words among a list of pseudowords.

Table 1. Demographic Information

Younger Adults Older Adults

\begin{tabular}{lll}
\hline Demographics & & \\
$n$ & 30 & 30 \\
Age range & $21-31$ & $60-71$ \\
Age $(M \pm S D)$ & $24.3(3.1)$ & $63.5(2.7)$ \\
Male/Female & $15 \mathrm{M} / 15 \mathrm{~F}$ & $15 \mathrm{M} / 15 \mathrm{~F}$ \\
Years of education $(M \pm S D)$ & $16.2(3.0)$ & $15.0(3.5)$ \\
\hline
\end{tabular}


Data on these two tests are missing for two older participants and one younger participant due to technical failure. For the remaining 57 participants, we computed independent samples $t$ tests to test for age differences in the Digit Symbol Test and the Spot-a-Word test. Younger adults $(M=63.97, S D=11.17)$ had higher scores than older adults $(M=49.25, S D=10.02)$ in the Digit Symbol Test $[t(53)=5.02, p<.01]$. In the Spot-a-Word Test, age groups differed substantially in terms of accuracy $[t(55)=$ $-2.87, p<.01$ ], with older subjects showing higher performance than younger adults $(M$ young $=0.60, S D=0.15$; $M$ old $=0.73, S D=0.18$ ). The observed age dissociation, with an age-related decrease in processing speed and an improvement in verbal knowledge across age, is in good agreement with the literature (e.g., Park et al., 2002; Lindenberger, Scherer, \& Baltes, 2001; Lindenberger \& Baltes, 1997).

\section{Task}

We used a letter $n$-back task during which participants saw a series of letters and had to compare the currently presented letter with the one they had seen one, two, or three letters earlier. For each letter, participants had to indicate by button press whether the letter they currently saw was the same as the comparison letter (for timing details, see Figure 1A). This task requires participants not only to hold task-relevant information on-line but also to constantly update WM and to conduct an on-line comparison between a stored and a currently presented item.

Crucially, based on behavioral pilot studies, the $n$-back task was optimized to test older adults. In studies using the $n$-back task with young adults, load level typically changes from one block to the other. Older adults' response times, however, are known to be slower in situations that require switching between task conditions (Kray \& Lindenberger, 2000). To decrease switch costs in the present study, we increased the duration of each block to 30 sec and used fixation blocks with durations of $20 \mathrm{sec}$. Also, we increased the homogeneity of block order by presenting two blocks of the same condition in direct succession (e.g., -2-2-1-1-3-3-).

\section{Testing Procedure}

Prior to entering the scanner, participants were asked to fill in a form on demographic information. Next, they were given verbal instructions about the $n$-back task and then practiced it for up to three runs. During practice they received feedback about their task performance. The Spota-Word and Digit Symbol Tests were administered in a separate testing session prior to the imaging session.

\section{MRI Data Acquisition}

Whole-brain MRI data were collected with a 1.5-T Siemens Vision MRI system (Erlangen, Germany). Functional data were acquired using an echo-planar imaging sequence with standard parameters $(\mathrm{TR}=2500 \mathrm{msec}, \mathrm{TE}=40 \mathrm{msec}$, flip angle $=90^{\circ}$, voxel size $=4 \times 4 \times 4 \mathrm{~mm}, 26$ slices acquired in ascending order approximately axial to the bicommissural plane, interslice gap $=0.15$ ). Each of the three experimental runs was preceded by three dummy volumes to achieve a steady state of tissue magnetization. The runs lasted about $5 \mathrm{~min}$. We acquired two structural scans: one proton-density-weighted sequence $(\mathrm{TR}=4350 \mathrm{msec}, \mathrm{TE}=$ 15 msec, flip angle $=180^{\circ}$, matrix $=252 \times 256$, voxel size $=$ $1 \times 1 \times 4 \mathrm{~mm}$ ), acquired in the same orientation as the functional EPI sequences for coregistration of the functional images, and one sagittally oriented high-resolution T1-weighted sequence $(\mathrm{TR}=20 \mathrm{msec}, \mathrm{TE}=5 \mathrm{msec}$, flip angle $=30^{\circ}$, matrix $=256 \times 256$, voxel size $=1 \times 1 \times$ $1 \mathrm{~mm})$.

\section{Data Analysis}

\section{Behavioral Data}

Behavioral data were analyzed with analyses of variance (ANOVAs) using SPSS for Windows 15.0 (SPSS, Chicago, IL). Repeated measures ANOVAs were conducted with age (young, old) as a between-subjects factor and task load (1-back, 2-back, and 3-back) as a within-subjects factor. Analyses were conducted separately for accuracy and response times. For the analysis of response times, trials below 200 msec were discarded and only correct responses were included in the analysis.

We also conducted an extreme-group analysis (cf. Nagel et al., 2009). The methods, results, and discussion related to this analysis are reported as supplementary information (SI).

\section{MRI Data}

MRI data were analyzed using a mixed-effects approach within the framework of the general linear model as implemented in FSL 4.1 (FMRIB's Software Library; www.fmrib.ox. ac.uk/fsl; Smith et al., 2004). Slice-time and motion-corrected volumes were spatially smoothed using a Gaussian kernel of $8 \mathrm{~mm}$ full width at half maximum and high-pass filtered $(\sigma=108 \mathrm{sec})$. Intrinsic autocorrelations were modeled using prewhitening. We used three regressors to model each of the three load conditions (1-back, 2-back, and 3-back) and a fourth regressor to model the appearance of the cue indicating the condition before each block. Regressors were convolved with a hemodynamic response function (gamma variate). Registration to high-resolution and standard images was carried out using FLIRT (Jenkinson, Bannister, Brady, \& Smith, 2002; Jenkinson \& Smith, 2001). Contrast images were computed for each condition per subject. They were then spatially normalized and transformed into standard space (Jenkinson et al., 2002). Group effects were computed using the transformed contrast images in a mixed-effects model treating subjects as random. We used a threshold of $Z>3.1(p<.001$, minimum cluster size $=$ 20 voxels) for whole-brain analyses. 


\section{Voxel-based Analysis: WM Network and Change in Activation with Load}

The contrast comparing the hardest task condition (3back) with fixation baseline was computed separately in younger and older adults to reveal the WM network. Furthermore, we investigated the effect of WM load by comparing 3-back with 1-back in both age groups. The Age $\times$ Load interaction was tested by contrasting the load-effect maps of younger and older adults. Even though the 3-back versus 1-back contrast is a better indicator of regions clearly involved in WM, it is also important to consider the comparison of 3-back to fixation baseline because this contrast shows that older adults' regions within the WM network are also activated at 3-back. This does not show up in the parametric contrast as, on average, older adults did not show an increase of activation across load.

We conducted two kinds of analyses to investigate whether age differences in functional activation are modulated by performance, an ROI analysis, and a psychophysiological interaction (PPI) analysis.

\section{ROI Analysis}

The first analysis aimed at testing changes in the BOLD signal of the different age groups in task-relevant regions. We conducted an ROI analysis, with regions in bilateral FPC, DLPFC, VLPFC, SMA, PMC, and PPC. These ROIs were defined based on functional activation during task performance. Within each age group, we computed a contrast comparing WM task-related activation (mean of 1-back, 2-back, and 3-back) to fixation baseline. Based on these contrast maps of each age group, we computed a conjunction map ( $p<.005$, uncorrected). As a result, the conjunction image showed activation in regions in which both younger and older adults showed significant activation at all load levels. By using this conjunction image, we avoid the problem of biasing the selection of voxels with regard to the load-related modulation that is of interest here (e.g., nonindependence errors; cf. Kriegeskorte, Simmons, Bellgowan, \& Baker, 2009). Finally, we defined ROIs by placing an 8- $\mathrm{mm}$ sphere around the peak activation in these regions (for MNI coordinates of ROIs, see Table 2). From these ROIs, mean percent signal change was extracted per subject for the three load conditions separately for each ROI.

Repeated measures ANOVAs per ROI were calculated to test for Age $\times$ Load interaction contrasts, that is, whether changes in the BOLD signal with load differed between age groups and whether this difference followed a linear pattern, a quadratic pattern, or both. To identify the course of the dose-response function, we first computed repeated measures ANOVAs separately for each age group and ROI to test whether polynomial contrasts of different load conditions revealed load-dependent changes in the BOLD signal. Taking the extreme group approach, we also examined dose-response functions in high- versus low-performing older adults (for details, see SI).
Table 2. Locations of ROI

\begin{tabular}{lcccr}
\hline & & \multicolumn{3}{c}{ MNI Coordinates } \\
\cline { 3 - 5 } ROI & $B A$ & $x$ & $y$ & $z$ \\
\hline Left & 10 & -34 & 50 & 12 \\
FPC & $97 / 46$ & -42 & 32 & 24 \\
DLPFC & $45 / 44$ & -42 & 20 & 2 \\
VLPFC & 6 & -46 & 6 & 32 \\
PMC & 7 & -40 & -48 & 44 \\
PPC & & & & \\
& 10 & 28 & 50 & 18 \\
Right & $97 / 46$ & 42 & 32 & 30 \\
FPC & $45 / 44$ & 38 & 16 & 4 \\
DLPFC & 6 & 50 & 6 & 26 \\
VLPFC & 7 & 36 & -48 & 48 \\
PMC & PPC & & & \\
\hline
\end{tabular}

The regions were defined based on the conjunction image of young and old adults of all load levels.

To statistically compare responsivity of the BOLD signal to individual subjects' performance, we next computed difference scores (delta scores) of the signal change for 3-back minus the signal change for 1-back based on the extracted values of percent signal change. These scores were $Z$-transformed and corrected for outliers (values deviating more than 2SDs from the grand average were excluded). Hierarchical regression analyses were conducted to test whether delta accounted for variance in accuracy beyond age group. Age group and then delta were entered as predictor variables with accuracy at 3-back as dependent variable.

\section{Psychophysiological Interaction Analysis}

To investigate the relation of responsivity in connectivity to performance, we conducted a PPI analysis, which provides a measure of effective connectivity and reveals how the coupling of a seed region and any other voxel in the brain changes with task condition (Rogers, Morgan, Newton, \& Gore, 2007; Friston et al., 1997). To conduct the PPI analysis, we set up a GLM analysis with three regressors: The first regressor was the demeaned time course from the seed ROI in left DLPFC, the second regressor represented WM load (3-back minus 1-back), and the third regressor was the vector product of the first two regressors, representing the psychophysiological interaction. Placing the PPI seed in left DLPFC was guided by a priori consideration reflecting the central role of DLPFC in the WM network in general, and during the $n$-back performance, in particular (Mattay et al., 2006; Callicott et al., 1999; Rypma \& D’Esposito, 1999; Rypma et al., 1999; Barch et al., 1997; Braver et al., 1997; 
Jonides et al., 1997; Klingberg et al., 1997; Manoach et al., 1997). We computed activation maps thresholded at $Z=2.6$ (corresponding to $p<.005$, minimal cluster size: 20) for the groups of younger and older adults. Significant activation in the case of the PPI analysis depicts in which voxels does the coupling with left DLPFC change with load. In a PPI whole-brain analysis, we also compared load-dependent change in connectivity in the extreme groups (high- and low-performing younger and older adults; see SI).

To test how changes in coupling were related to behavioral outcomes (accuracy), we defined ROIs based on the PPI results for the younger adults. We masked the activation PPI result of young adults with the functionally defined WM network, based on the conjunction across age groups of the 3-back condition minus fixation. We thereby aimed to constrain the ROI analysis to the core WM network. ROIs in right DLPFC (MNI 50, 26, 30), left SMA (MNI $-8,6,54)$, left PMC (MNI $-30,4,50)$, and right PPC (MNI $36,-36,46)$ were defined by drawing an $8-\mathrm{mm}$ sphere around peak PPI results in these regions. From the ROIs, we extracted the beta estimate for the PPI regressor for each subject, indicating the change in coupling of these regions with left DLPFC across load. We $Z$-transformed this PPI score, and computed a hierarchical regression analysis entering age group and then PPI score as predictor variables of 3-back accuracy.

\section{RESULTS}

\section{$\boldsymbol{n}$-back Performance}

A repeated measures ANOVA revealed that main effects of age were significant for accuracy $[F(1,58)=21.77, p<$ $\left..01, \eta^{2}=.28\right]$ and correct response times $[F(1,58)=$ $11.86, p<.01, \eta^{2}=.17$ ], with younger adults being more accurate and faster than older adults. Similarly, the main effects of load were significant for accuracy $[F(2,116)=$
91.16, $\left.p<.01, \eta^{2}=.61\right]$ and correct response times $[F(2$, $\left.116)=89.37, p<.01, \eta^{2}=.61\right]$. The Age $\times$ Load interaction showed a trend toward significance for accuracy $\left[F(2,116)=2.37, p<.09, \eta^{2}=.03\right]$, and was significant for correct response times $[F(2,116)=6.85, p<.01$, $\left.\eta^{2}=.11\right]$, with older adults showing greater decline with increasing load than younger adults (see Figure 1B; for results of the extreme group analysis, see SI).

\section{fMRI}

\section{Whole-brain Analysis}

Verbal WM network in younger and older adults. Contrasting 3-back with fixation baseline revealed a frontoposterior network including FPC, DLPFC and VLPFC, SMA, PMC, and PPC. Notably, younger and older adults activated largely overlapping networks (see Figure 2A).

Load effect and Age $\times$ Load interaction. We tested the effect of load by comparing the BOLD response at 3-back to 1-back in both age groups (for peak activations, see Figure $2 \mathrm{~B}$ and Table 3). Activation increased with load in younger, but not in older, adults in DLPFC, VLPFC, PMC, and ventral PPC, resulting in significant Age $\times$ Load interactions (see Figure 2C).

\section{ROI Analysis}

The mean percent signal change extracted from each ROI and for each subject was analyzed statistically: A repeated measures ANOVA testing the Age $\times$ Load interaction revealed significant linear interaction contrasts in several ROIs, reflecting the linear increase of BOLD signal with load in younger, but not in older, adults. Specifically, interaction contrasts were reliable in left FPC $[F(1,58)=7.11, p<$ $.05, \eta^{2}=.11$ ], bilateral DLPFC [left DLPFC: $F(1,58)=$ $6.48, p<.05, \eta^{2}=.10$; right DLPFC: $F(1,58)=4.90, p<$

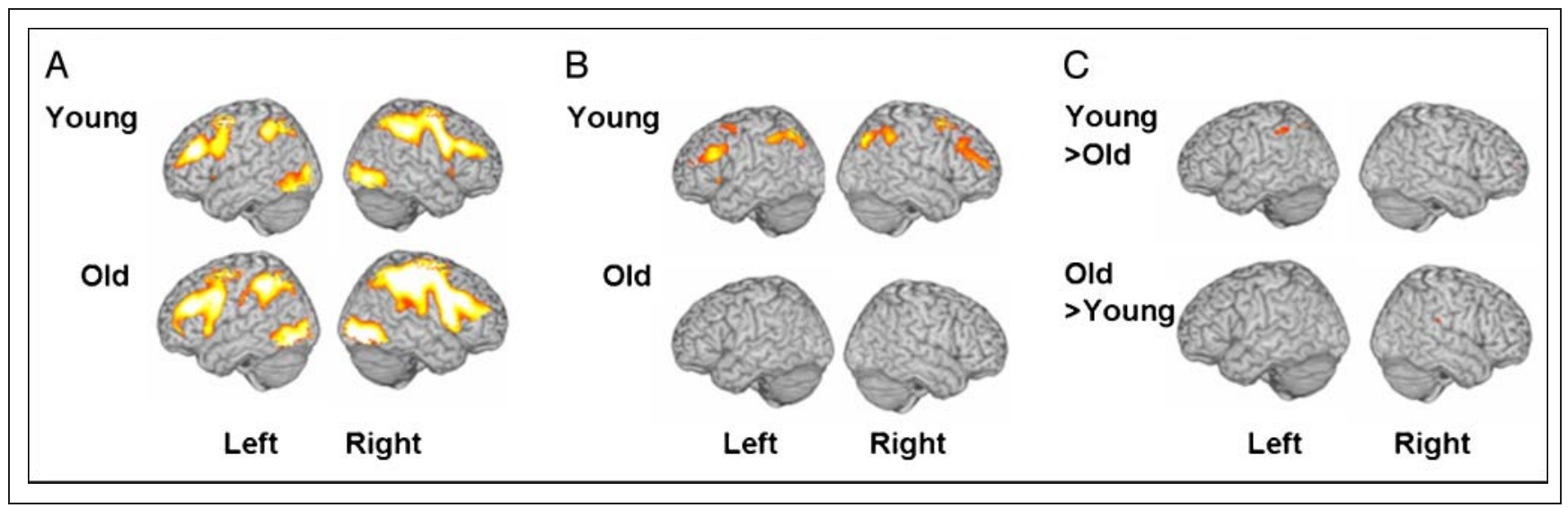

Figure 2. (A) The WM network in younger and older adults (3-back compared to fixation baseline; $Z>3.1$, corresponding to $p<.001$, cluster size threshold $=20$ ). Similar activation patterns in younger and older adults. More extended activation in older adults in inferior frontal cortex and parietal cortex. (B) Load effect in young and older adults $(Z>3.1$, cluster size threshold $=20)$ and $(C)$ Load by Age interaction. Increase of activation with load in younger but almost not in older adults (3-back compared to 1-back; $Z=3.1$; cluster size threshold $=20$ ). 
Table 3. Peak Activation of the Whole-brain Contrast 3-Back versus 1-Back in Young and Older Adults (Z > 3.1; Min Cluster Size $=20$ )

\begin{tabular}{|c|c|c|c|c|c|c|c|}
\hline \multirow[b]{2}{*}{ Hemisphere } & \multirow[b]{2}{*}{ Region } & \multirow[b]{2}{*}{$B A$} & \multirow[b]{2}{*}{ Voxel } & \multirow[b]{2}{*}{$Z-\operatorname{Max}$} & \multicolumn{3}{|c|}{ MNI Coordinates } \\
\hline & & & & & $x$ & $y$ & $z$ \\
\hline \multicolumn{8}{|l|}{ Young } \\
\hline Right & SMA & 6 & 13,049 & 6.23 & 8 & 22 & 42 \\
\hline Right & PPC & 7 & 6446 & 5.93 & 28 & -68 & 52 \\
\hline Right & VLPFC & 45 & 472 & 5.35 & 36 & 24 & -2 \\
\hline Right & Lingual gyrus & & 72 & 3.63 & 4 & -82 & -18 \\
\hline Left & Middle temporal gyrus & 21 & 36 & 3.64 & -56 & -50 & -10 \\
\hline \multicolumn{8}{|l|}{ Old } \\
\hline Left & SMA & 6 & 36 & 3.22 & -18 & 8 & 60 \\
\hline Right & Precuneus & & 23 & 3.09 & 8 & -70 & 44 \\
\hline Left & (para-) Cingulate gyrus & 32 & 23 & 3.14 & -6 & 34 & 24 \\
\hline
\end{tabular}

$\left..05, \eta^{2}=.08\right]$, SMA $\left[F(1,58)=7.20, p<.01, \eta^{2}=.11\right]$, bilateral PPC [left PPC: $F(1,58)=4.79, p<.05, \eta^{2}=.08$; right PPC: $\left.F(1,58)=4.36, p<.05, \eta^{2}=.07\right]$, and at trend level in left PMC $\left[F(1,58)=3.02, p<.10, \eta^{2}=.05\right]$. There were no significant effects for the quadratic interaction contrasts.

To follow up on these interactions, we computed repeated measures ANOVAs testing the load contrasts separately for each age group. In younger, but not older, adults, activation increased linearly up to the highest load level (3-back) in all ROIs except right VLPFC (see Figure 3). Specifically, in younger adults, the BOLD response increased significantly in bilateral FPC [left FPC: $F(1,29)=11.84$, $p<.01, \eta^{2}=.29$; right FPC: $F(1,29)=5.25, p<.05, \eta^{2}=$ .15], bilateral DLPFC [left DLPFC: $F(1,29)=11.69, p<$ $.01, \eta^{2}=.29$; right DLPFC: $F(1,29)=9.60, p<.01$, $\left.\eta^{2}=.25\right]$, left VLPFC $\left[F(1,29)=4.52, p<.05, \eta^{2}=\right.$ $.14]$, SMA $\left[F(1,29)=8.39, p<.01, \eta^{2}=.22\right]$, right PMC $\left[F(1,29)=5.32, p<.05, \eta^{2}=.16\right]$, bilateral PPC [left PPC: $F(1,29)=10.52, p<.01, \eta^{2}=.27$; right PPC: $F(1$, $\left.29)=4.78, p<.05, \eta^{2}=.14\right]$, and at trend level in left PMC $\left[F(1,29)=3.69, p<.10, \eta^{2}=.11\right]$. By contrast, for older adults, there was a linear increase in left FPC only. There were no significant load or Load $\times$ Age effects for quadratic contrasts (for results of the extreme group analysis, see SI).

\section{Regression: Load-dependent BOLD Signal Change Predicts $n$-back Task Performance}

To test whether BOLD responsivity to WM challenge is related to WM performance, we created an index of re- sponsivity by subtracting the signal change at the 1-back condition from that of the 3-back condition (outlier correction: exclusion of values deviating more than $2 S D$ s from the grand mean; depending on the ROI, 2-4 subjects were excluded). We performed hierarchical regression analyses using age group, delta, and their interaction term as factors to test whether delta accounted for variance in accuracy beyond age. The regressions reported here were computed separately for each ROI. Results that remain statistically reliable after correcting for the false discovery rate (FDR) are indicated by an asterisk. The change statistics $\left(R_{\text {change }}^{2}\right)$ were significant in left PMC $\left[R_{\text {change }}^{2}=.085, F_{\text {change }}(1 / 55)=\right.$ $6.12, p=.01]$, right PMC $\left[R_{\text {change }}^{2}=.13, F_{\text {change }}(1 / 53)=\right.$ $\left.9.84, p=.003^{*}\right]$, and right PPC $\left[R_{\text {change }}^{2}=.06, F_{\text {change }}(1)\right.$ $54)=4.2, p=.05]$, and at trend level in left DLPFC $\left[R_{\text {change }}^{2}=\right.$ $\left..042, F_{\text {change }}(1 / 55)=2.76, p=.1\right]$ and right DLPFC $\left[R_{\text {change }}^{2}=\right.$ $\left..04, F_{\text {change }}(1 / 55)=2.71, p=.1\right]$, showing that delta beyond age group is an important predictor of $n$-back performance beyond age. The interaction term of age group and delta was not significant in any of the ROIs, except for a trendlevel significance in right PPC $(p=.09)$. Figure 4 displays the scatterplots illustrating the associations between delta and accuracy.

\section{PPI Analysis}

The PPI analysis revealed that the load-dependent changes in connectivity between left DLPFC and other brain regions differed between age groups. Younger adults showed changes in effective connectivity across load in key WM

Figure 3. This figure depicts BOLD signal changes in younger and older adults across load (younger adults: green; older adults: purple; lower task demand is represented by lighter colors). Change in BOLD signal increases monotonously in all ROIs except right VLPFC in younger but not in older adults $(* *$ reliable at $p<.01 ; *$ reliable at $p<.05 ;+$ trend $p=.051-.1$ ). In all ROIs reliable contrasts were linear (as indicated by the "L"); quadratic contrasts were not significant. ROI locations are shown in blue. 


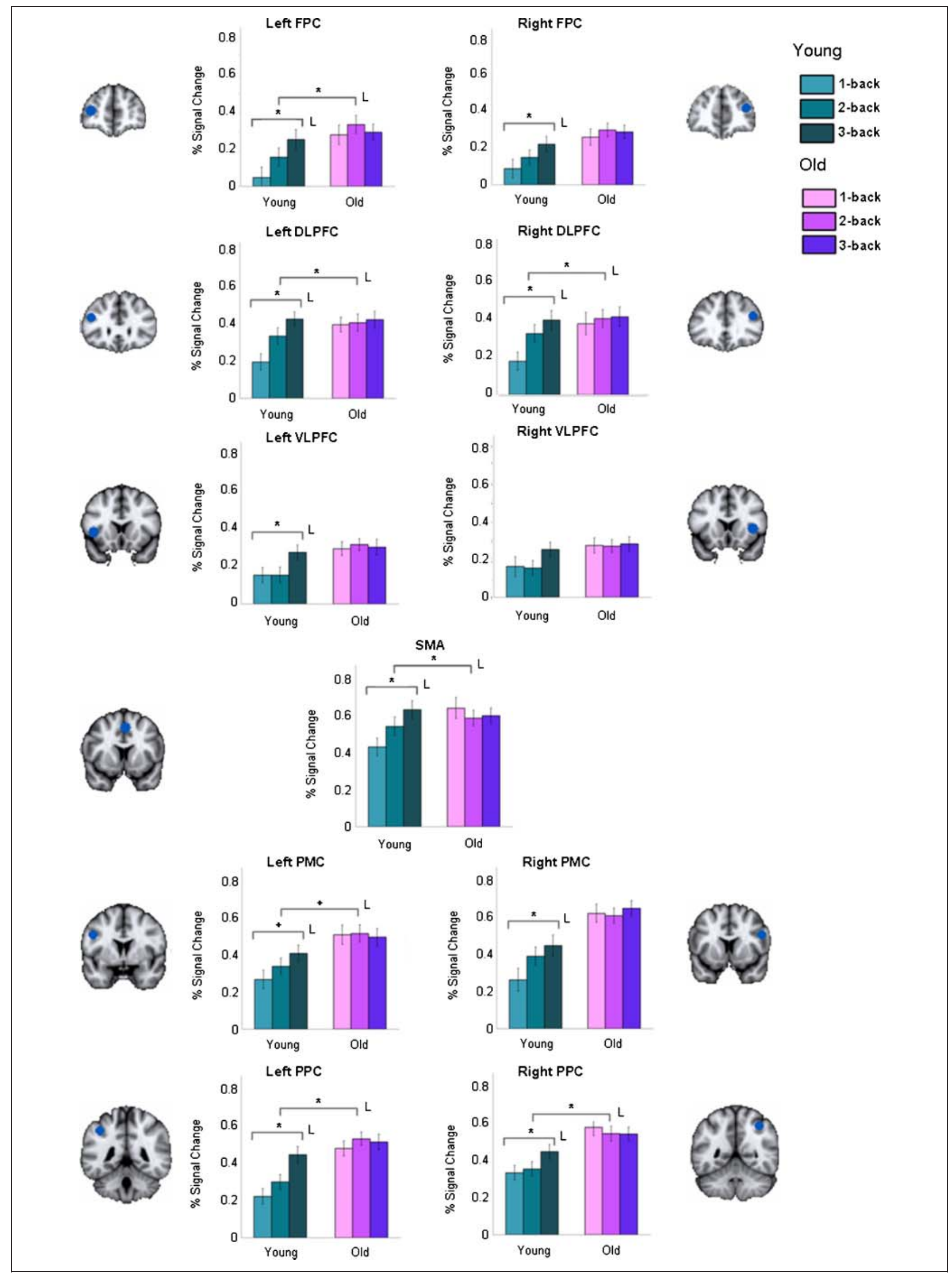


regions including bilateral $\mathrm{PFC}$, SMA, left $\mathrm{PMC}$ and right PPC, whereas older adults showed load-dependent changes in coupling only in left anterior frontal cortex and temporal regions (see Figure 5 and Table 4; for results of the extreme group analysis, see SI).

To test whether changes in coupling predict WM task performance, we computed a hierarchical regression using age group and the PPI score as predictors and accuracy at 3-back as dependent variable revealed a trend-level load-dependent change in connectivity between left PMC $\left[R_{\text {change }}^{2}=.051, F_{\text {change }}(1 / 57)=3.554, p=.064\right.$, uncorrected] and left DLPFC, which accounted for variance in accuracy beyond age group (see Figure 6). Again, the interaction term was not significant.

\section{DISCUSSION}

Using a verbal $n$-back task with three load levels, this study investigated individual differences in the local and distributed brain responses of younger and older adults in relation to a WM challenge. We conducted two sets of analyses. First, we investigated dose-response functions, derived by extracting percent signal change from ROIs in task-relevant regions (bilateral FPC, DLPFC, VLPFC, SMA, PMC, and PPC) for each age group and determined whether changes in the BOLD response from 1-back to 3-back (delta) predicted task performance. Next, we investigated load-dependent changes in the coupling between brain regions with a PPI analysis, using left DLPFC as seed region, and tested whether connectivity scores predicted performance.

\section{Responsivity of BOLD Activation to Changing Task Difficulty Is Related to Performance}

The ROI analysis yielded several important findings. By examining dose-response functions averaged across each age group, we found, in accordance with prior research (Cappell et al., 2010; Schneider-Garces et al., 2010; Nyberg et al., 2008; Mattay et al., 2006), that dose-response functions differed between younger and older adults (see Figure 3). Younger adults showed an increased BOLD response to a WM challenge, whereas the corresponding response in older adults was compromised. Note that in younger adults the BOLD response did not level off, suggesting that they did not reach their capacity limit at the 3-back condition. These results can be interpreted within the CRUNCH framework (Reuter-Lorenz \& Cappell, 2008): Due to agerelated neurobiological declines, BOLD responsivity is compromised. This may be due to a CRUNCH-like mechanism, in the sense that older individuals with neurobiological decline who show compensatory overactivation at low load cannot increase activation at high load.

When examining individual differences in dose-response functions by taking the delta score (the increase of acti- vation from load one to three) of each person and relating it to individual variation in WM performance at 3-back, we found that local BOLD responsivity predicts WM performance. This is also apparent from the extreme-group analysis reported in the SI. These individual differences in responsivity would go unnoticed if analyses were restricted to mean differences between age groups. The regression analysis revealed that delta entered into the equation after age accounted for significant amounts of variance in accuracy at 3-back in bilateral PMC and right PPC and, at trend level, in bilateral DLPFC (see Figure 4), suggesting that prefrontal and parietal modulation is important to task performance. Although the group average of the dose-response function for older adults (Figure 3) was rather flat in most ROIs, the regressions and the extreme-group analysis show that individual differences in the brain's responsivity to increasing task demands contribute to individual differences in accuracy. In terms of the CRUNCH account, we found individual differences in the point where maximum BOLD activation is reached, particularly among older adults. Thus, there are individual differences in the range of responsivity at which people operate and these are predictive of individual variation in behavioral outcome. These results should not be interpreted to mean that there is generally no responsivity in older adults, or that there is perfect linear responsivity in the young. Instead, the results reported here show that responsivity is compromised if levels of task difficulty are high, given an individual's neurobiological resources.

Although older adults may show intact responsivity at low task load, it is important, both conceptually and from an applied perspective, to investigate how older adults function in cognitively demanding situations. In the present study, performance in older adults was not at chance level. Although older adults did not reach a behavioral floor, at least some older adults showed a decrease in BOLD activation already at 2-back, suggesting that they reached an activation limit. Reaching such an activation limit may precede reaching a behavioral floor.

Compensation may be necessary and adaptive in the face of neurobiological decline. Compensation, however, comes with a cost. Overactivation may be helpful at low load but makes further increase of activation at high load difficult (Reuter-Lorenz \& Cappell, 2008). Also, as can be seen from our results, in some older adults, responsivity is similar to young adults. In agreement with similar results reported by Nagel et al. (2009), this finding suggests that some older adults are able to maintain a youth-like pattern of activation that does not require compensatory overactivation at low load in the first place. Longitudinal studies are needed to identify the antecedents and correlates of successfully preserved WM functioning in old age. To the extent that the relevant mechanisms can be manipulated, the resulting findings may inform interventions that foster successful WM aging. 


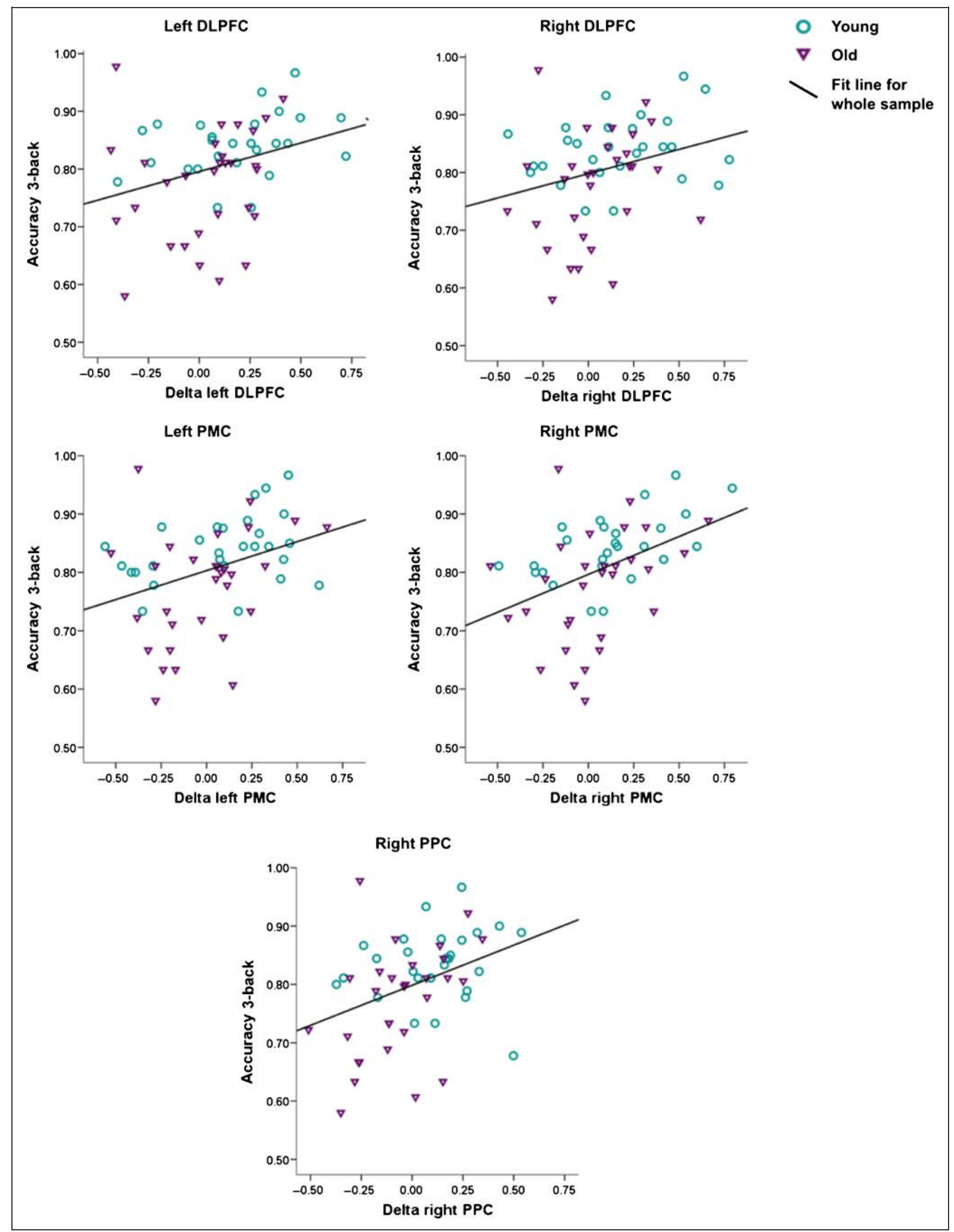

Figure 4. Delta (BOLD signal change 3-back minus 1-back, Z-standardized) predicts accuracy at 3-back in bilateral PMC, right PPC and at trend level in bilateral DLPFC. This suggests that responsivity of PMC to WM task demands is of importance for optimal WM performance. 


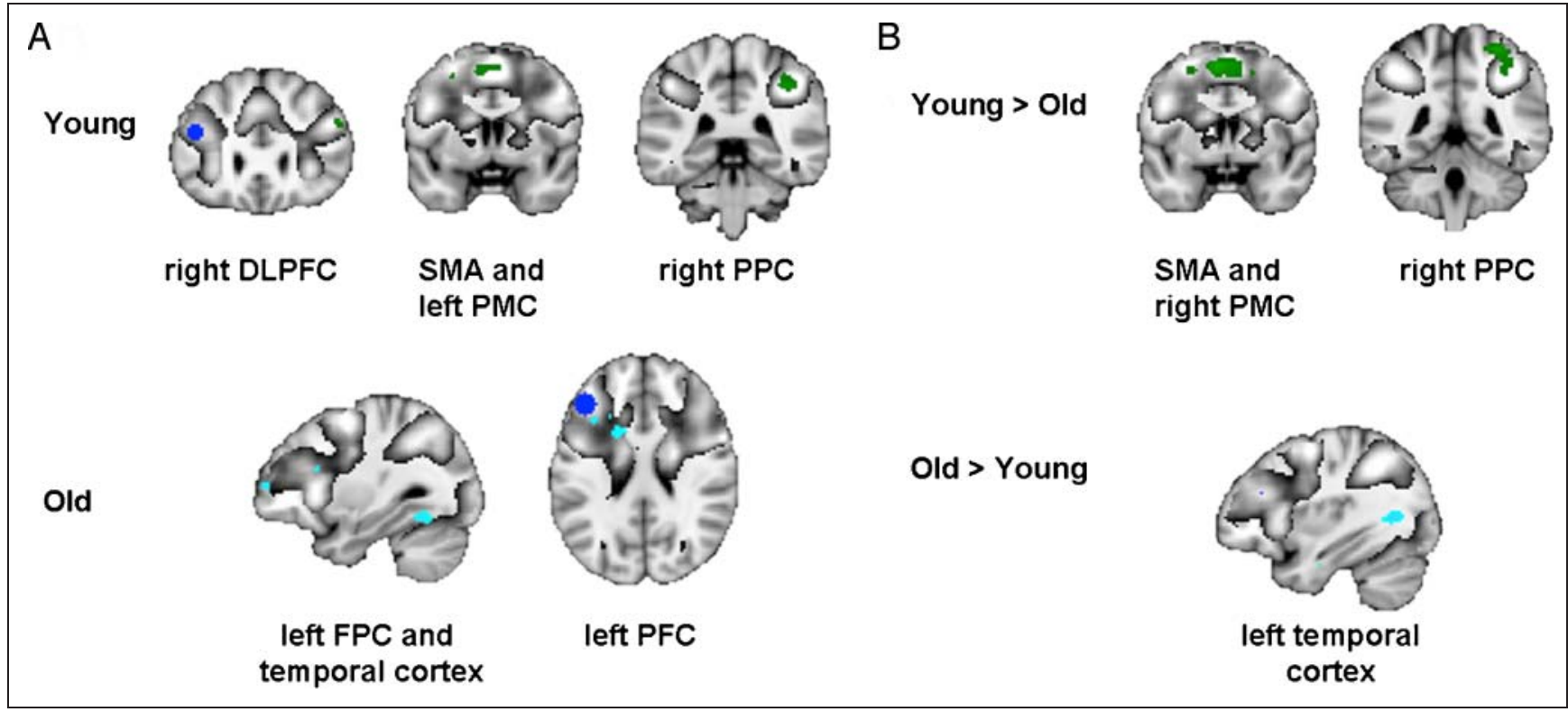

Figure 5. Load-dependent change in coupling between left DLPFC seed and other brain regions overlaid on WM network: WM network (conjunction of 3-back compared to fixation, young and old, $Z>2.6$ ) is depicted in gray, the left DLPFC seed for PPI analysis is marked by a blue point. A: brain regions showing a load-dependent change in coupling with left DLPFC in young participants are displayed in green (right DLPFC, SMA, and right PPC). Lower left side: brain regions showing a load-dependent change in coupling with left DLPFC in older participants are displayed in turquoise (left FPC, left PFC, and left temporal cortex). B: regions where the change in coupling is greater in young adults (SMA, PMC, and PPC). Lower right side: regions where change in coupling is greater in older adults (left temporal cortex).

\section{Adjustment of Connectivity within the WM Network to Changing Task Difficulty Predicts Performance}

In the PPI analysis, we investigated the adjustment of connectivity within the WM network to changing task demands in younger and older adults. Younger adults' coupling in core regions of the fronto-parietal WM net- work changed with load (right DLPFC, SMA, left PMC, and right PPC; see Figure 5). This finding is in accordance with prior research on load-related change in coupling in younger adults (Axmacher et al., 2008; Chang, CrottazHerbette, \& Menon, 2007; Narayanan et al., 2005; Honey et al., 2002). Interestingly, the groups of younger and older adults differed, on average, in these connectivity patterns. Contrary to the young, older adults showed only a

Table 4. Location of Peak Activation in PPI Analysis for Younger and Older Adults $(Z>2.6)$

\begin{tabular}{|c|c|c|c|c|c|c|c|}
\hline \multirow[b]{2}{*}{ Hemisphere } & \multirow[b]{2}{*}{ Region } & \multirow[b]{2}{*}{$B A$} & \multirow[b]{2}{*}{ Voxel } & \multirow[b]{2}{*}{$Z$-Max } & \multicolumn{3}{|c|}{ MNI Coordinates } \\
\hline & & & & & $x$ & $y$ & $z$ \\
\hline \multicolumn{8}{|l|}{ Young } \\
\hline Left & SMA & 6 & 1039 & 3.2 & -10 & 6 & 52 \\
\hline Right & PPC & 7 & 670 & 3.44 & 40 & -40 & 44 \\
\hline Right & DLPFC & 9 & 162 & 3.22 & 50 & 26 & 30 \\
\hline \multicolumn{8}{|l|}{ Old } \\
\hline Left & VLPFC & $45 / 44$ & 3239 & 2.95 & -34 & 20 & 22 \\
\hline Left & Thalamus & $23 / 30$ & 2723 & 3.32 & -2 & -34 & 6 \\
\hline Right & Lingual gyrus & & 731 & 2.6 & 2 & -80 & -20 \\
\hline Right & Inferior temporal gyrus & 37 & 504 & 2.88 & 54 & -46 & -12 \\
\hline Right & Occipital pole & $17 / 18$ & 216 & 2.65 & 10 & -100 & -4 \\
\hline
\end{tabular}


Figure 6. Task-dependent change in coupling between left DLPFC and left PMC ( $z$-standardized) predicts accuracy at 3-back. This suggests that the coupling between left DLPFC and left PMC is important for good performance in the $n$-back task in both younger and older adults.

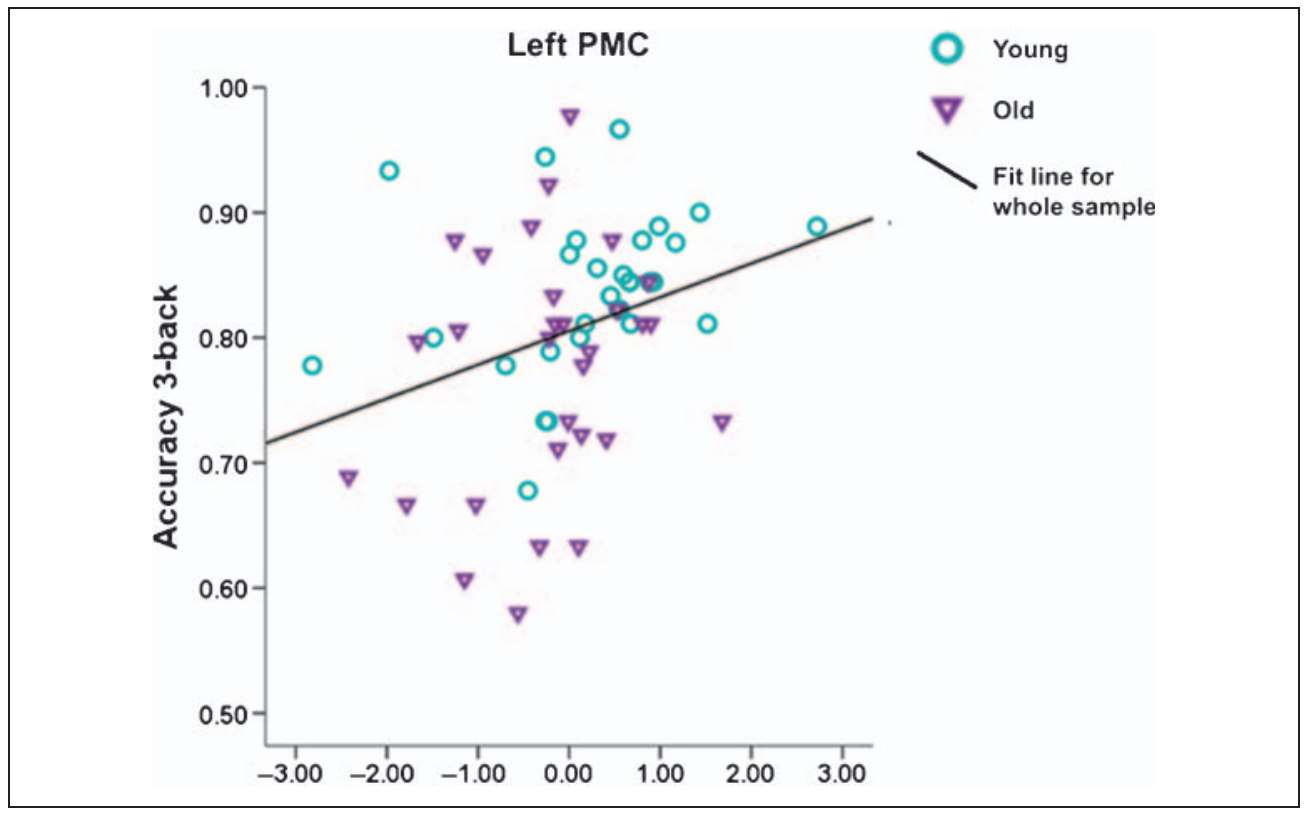

load-dependent change in coupling with left DLPFC in left FPC, and in regions that are not part of the WM network, namely, medial PFC and inferior temporal visual regions. The fact that the PPI analysis did not yield any overlap in results for younger and older adults is not surprising because the PPI analysis only captures regions in which connectivity with left DLPFC changes with load. Hence, the observed age differences indicate that the effective connectivity of the WM network differs, on average, between early and late adulthood.

The present study provides novel information about individual differences in WM by showing that variation in load-dependent connectivity adjustments with left DLPFC predict individual differences in WM performance at high load. Although only at a trend level and not corrected for multiple comparisons, we found that the PPI index predicted accuracy at 3-back in left PMC (see Figure 6). Thus, individuals with more flexible adjustment of the connection between left DLPFC and PMC performed better, suggesting that adjustment of this connection contributes to WM performance. The whole-brain extremegroup PPI analysis reported in the SI provides converging evidence for individual differences in load-dependent change in connectivity. There were differences in PPI responsivity within both age groups. Further, it was apparent that only the old low performers showed no change in connectivity across load whatsoever within the WM network.

Note, however, that also old low performers might have shown a change in connectivity in less demanding tasks. In the present task, adjustment of connectivity to task demand was compromised in many older adults, which could be due to at least two reasons. First, for those individuals where local BOLD responsivity was low, no PPI change can be expected. Thus, individuals who reached their
BOLD limit at low load in various ROIs are very unlikely to show increased connectivity between those ROIs at high load. Second, age-related decline in gray- and whitematter integrity could have directly affected the coordinated processing within the WM network (Bennett et al., 2001).

Taken together, the results of the present study provide new insights into individual differences in WM performance at the neural level. As hypothesized, we found that individuals with higher responsivity of both local functional brain activation as well as the connectivity between regions of the WM network performed better in the WM task. These results are in accordance with earlier studies reporting that the responsivity of the WM network in older adults as a group is compromised relative to younger adults (e.g., Cappell et al., 2010; Schneider-Garces et al., 2010; Mattay et al., 2006). However, our findings add the important qualification that differences in responsivity continue to predict individual differences in task performance after age group differences in performance have been statistically controlled. The results reported here show how individual differences in the load-dependent BOLD response of younger and older adults relate to individual differences in WM performance. An important next step is to delineate specific WM mechanisms that explain individual differences in the responsivity of the WM network, and their connection to age-related brain changes. Still, the results of this study suggest that a substantial portion of the local and global network characteristics that permit individuals in the adult age range to adequately respond to WM challenges is age-invariant (cf. Cappell et al., 2010; Nagel et al., 2009). From this perspective, normal aging reduces the likelihood of an adequately responsive WM network, due to factors that are more or less similar to the reasons that bring about individual differences in WM performance in 
age-homogeneous samples. Some of these factors are discussed next.

\section{Possible Factors Affecting Responsivity}

Individual differences in responsivity may be associated with variation in brain anatomy (i.e., cortical thickness, vasculature, myelination). For instance, age-related decline in white-matter integrity may limit the coupling between brain regions with increasing load. Several studies show that white-matter tracts, particularly in PFC, undergo age-related changes (Burzynska et al., 2010; Madden, Bennett, \& Song, 2009; O’Sullivan et al., 2001; Gunning-Dixon \& Raz, 2000; Pfefferbaum et al., 2000), which contribute to changes in WM performance. Furthermore, there are marked agerelated changes in the vascular system that likely affect BOLD responsivity (Gazzaley \& D'Esposito, 2005). Responsivity might also be affected by dopamine functions. This neurotransmitter regulates processing in PFC and other brain regions (e.g., Meyer-Lindenberg et al., 2006), whereby higher levels of dopamine are typically related to more efficient processing (Meyer-Lindenberg et al., 2006; Li \& Sikström, 2002; Servan-Schreiber, Printz, \& Cohen, 1990). Dopamine also affects the coupling between brain regions (Ito \& Schuman, 2008; Nagano-Saito et al., 2008). Age-related dopamine losses (Kaasinen et al., 2000; Suhara et al., 1991) may thus influence both the local BOLD response (Bäckman, Linderberger, Li, \& Nyberg, 2010; Li \& Sikström, 2002) and the coupling of WM-relevant brain regions. Individual differences in neurobiological aging trajectories in terms of onset and severity may lead to increased variation in responsivity, and thus, in cognitive performance among older adults (Bäckman et al., in press; Lindenberger \& Ghisletta, 2009; de Frias et al., 2007; Bäckman, Small, Wahlin, \& Larsson, 1999; Baltes \& Lindenberger, 1997).

In summary, the present study underscores the importance of the brain's responsivity to task demands for proficient WM functioning. We observed sizeable and performance-related individual differences in local and network-related adjustments to task demands. Across younger and older adults, higher responsivity of local activation (delta) and network connectivity (PPI) predicted better WM task performance at high levels of task difficulty. We conclude that the prevailing focus on comparing group averages comes at a double cost. This focus hides the heterogeneity of WM response patterns within the samples that are being contrasted, such as groups of younger and older adults. At the same time, the focus on average group differences also hides the notable degree of invariance in the brain characteristics that promote proficient WM performance across age groups (see also Nagel et al., 2009). WM functioning may be more heterogeneous (e.g., within groups) and more invariant (e.g., across groups) than commonly assumed. Documenting this heterogeneity in neural processing in relation to performance opens a window to delineating mechanisms that promote positive cognitive outcomes.

\section{Acknowledgments}

This research was supported by the Max Planck Society, including a grant from the innovation fund of the Max Planck Society (M.FE. A.BILD0002). It also was supported by a grant from the German Federal Ministry for Research to the Berlin NeuroImaging Center (01GO0501). L. B. was supported by the Swedish Research Council (521-2007-2892), Swedish Brain Power, and by an Alexander von Humboldt Research Award. I. E. N. was supported by a predoctoral fellowship of the International Max Planck Research School, The Life Course: Evolutionary and Ontogenetic Dynamics (LIFE). Parts of this article were written while U. L. was a fellow at the Center for Advanced Study in the Behavioral Sciences at Stanford University. We thank the technical assistants Kirsten Becker, Silke Becker, Anita Günter, and Karola Rockmann.

Reprint requests should be sent to Irene E. Nagel, Department of Education and Psychology, Freie Universität, Habelschwerdter Allee 45, 14195 Berlin, or via e-mail: irene.nagel@fu-berlin.de.

\section{REFERENCES}

Andrews-Hanna, J. R., Snyder, A. Z., Vincent, J. L., Lustig, C., Head, D., Raichle, M. E., et al. (2007). Disruption of large-scale brain systems in advanced aging. Neuron, 56, 924-935.

Axmacher, N., Schmitz, D. P., Wagner, T., Elger, C. E., \& Fell, J. (2008). Interactions between medial temporal lobe, prefrontal cortex, and inferior temporal regions during visual working memory: A combined intracranial EEG and functional magnetic resonance imaging study. Journal of Neuroscience, 28, 7304-7312.

Babcock, R. L., \& Salthouse, T. A. (1990). Effects of increased processing demands on age differences in working memory. Psychology and Aging, 5, 421-428.

Bäckman, L., Karlsson, S., Fischer, H., Karlsson, P., Brehmer, Y., Rieckmann, A., et al. (in press). Dopamine $\mathrm{D}_{1}$ receptors and age differences in brain activation during working memory. Neurobiology of Aging.

Bäckman, L., Lindenberger, U., Li, S. C., \& Nyberg, L. (2010). Linking cognitive aging to alterations in dopamine neurotransmitter functioning: Recent data and future avenues. Neuroscience and Biobehavioral Reviews, 34, 670-677.

Bäckman, L., Nyberg, L., Lindenberger, U., Li, S. C., \& Farde, L. (2006). The correlative triad among aging, dopamine, and cognition: Current status and future prospects. Neuroscience and Biobehavioral Reviews, 30, 791-807.

Bäckman, L., Small, B. J., Wahlin, A., \& Larsson, A. (1999). Cognitive functioning in very old age. In F. I. Craik \& T. Salthouse (Eds.), Handbook of aging and cognition (Vol. 2, pp. 499-558). Mahwah, NJ: Erlbaum.

Baddeley, A. (2003). Working memory: Looking back and looking forward. Nature Reviews Neuroscience, 4, 829-839.

Baltes, P. B., \& Lindenberger, U. (1997). Emergence of a powerful connection between sensory and cognitive functions across the adult life span: A new window to the study of cognitive aging? Psychology and Aging, 12, $12-21$.

Barbas, H., \& Pandya, D. N. (1989). Architecture and intrinsic connections of the prefrontal cortex in the rhesus monkey. Journal of Comparative Neurology, 286, 353-375.

Barbas, H., \& Zikopoulos, B. (2007). The prefrontal cortex and flexible behavior. Neuroscientist, 13, 532-545.

Barch, D. M., Braver, T. S., Nystrom, L. E., Forman, S. D., Noll, D. C., \& Cohen, J. D. (1997). Dissociating working 
memory from task difficulty in human prefrontal cortex. Neuropsychologia, 35, 1373-1380.

Bennett, P. J., Sekuler, A. B., McIntosh, A. R., \& Della-Maggiore, V. (2001). The effects of aging on visual memory: Evidence for functional reorganization of cortical networks. Acta Psychologica (Amsterdam), 107, 249-273.

Braver, T. S., Cohen, J. D., Nystrom, L. E., Jonides, J., Smith, E. E., \& Noll, D. C. (1997). A parametric study of prefrontal cortex involvement in human working memory.

Neuroimage, 5, 49-62.

Burzynska, A., Preuschhof, C., Bäckman, L., Nyberg, L., Li, S.-C., Lindenberger, U., et al. (2010). Age-related differences in white-matter microstructure: Region-specific patterns of diffusivity. Neuroimage, 49, 2104-2112.

Cabeza, R., Anderson, N. D., Locantore, J. K., \& McIntosh, A. R. (2002). Aging gracefully: Compensatory brain activity I high-performing older adults. Neuroimage, 17, 1394-1402.

Cabeza, R., McIntosh, A. R., Tulving, E., Nyberg, L., \& Grady, C. L. (1997). Age-related differences in effective neural connectivity during encoding and recall. NeuroReport, 8, 3479-3483.

Callicott, J. H., Mattay, V. S., Bertolino, A., Finn, K., Coppola, R., Frank, J. A., et al. (1999). Physiological characteristics of capacity constraints in working memory as revealed by functional MRI. Cerebral Cortex, 9, 20-26.

Cappell, K. A., Gmiendl, L., \& Reuter-Lorenz, P. A. (2010). Age differences in prefrontal recruitment during verbal working memory depend on memory load. Cortex, 46, 462-473.

Chang, C., Crottaz-Herbette, S., \& Menon, V. (2007). Temporal dynamics of basal ganglia response and connectivity during verbal working memory. Neuroimage, 34, 1253-1269.

Conway, A. R. A., Jarrold, C., Kane, M. J., Miyake, A., \& Towse, J. N. (Eds.) (2008). Variation in working memory. New York: Oxford University Press.

Cook, I. A., Bookheimer, S. Y., Mickes, L., Leuchter, A. F., \& Kumar, A. (2007). Aging and brain activation with working memory tasks: An fMRI study of connectivity. International Journal of Geriatric Psychiatry, 22, 332-342.

Croxson, P. L., Johansen-Berg, H., Behrens, T. E., Robson, M. D., Pinsk, M. A., Gross, C. G., et al. (2005). Quantitative investigation of connections of the prefrontal cortex in the human and macaque using probabilistic diffusion tractography. Journal of Neuroscience, 25, 8854-8866.

D'Esposito, M. (2007). From cognitive to neural models of working memory. Philosophical Transaction of the Royal Society of London, Series B, Biological Sciences, 362, 761-772.

de Frias, C. M., Lovden, M., Lindenberger, U., \& Nilsson, L. G. (2007). Revisiting the dedifferentiation hypothesis with longitudinal multi-cohort data. Intelligence, 35, 381-392.

Damoiseaux, J. S., Beckmann, C. F., Arigita, E. J., Barkhof, F., Scheltens, P., Stam, C. J., et al. (2008). Reduced resting-state brain activity in the "default network" in normal aging. Cerebral Cortex, 18, 1856-1864.

Della-Maggiore, V., Sekuler, A. B., Grady, C. L., Bennett, P. J., Sekuler, R., \& McIntosh, A. R. (2000). Corticolimbic interactions associated with performance on a short-term memory task are modified by age. Journal of Neuroscience, 20, 8410-8416.

Duverne, S., Motamedinia, S., \& Rugg, M. D. (2009). The relationship between aging, performance, and the neural correlates of successful memory encoding. Cerebral Cortex, 19, 733-744.
Friston, K. J., Buechel, C., Fink, G. R., Morris, J., Rolls, E., \& Dolan, R. J. (1997). Psychophysiological and modulatory interactions in neuroimaging. Neuroimage, 6, 218-229.

Gazzaley, A., Cooney, J. W., Rissman, J., \& D'Esposito, M. (2005). Top-down suppression deficit underlies working memory impairment in normal aging. Nature Neuroscience, 8, 1298-1300.

Gazzaley, A., \& D'Esposito, M. (2005). BOLD functional MRI and cognitive aging. In R. Cabeza, L. Nyberg, \& D. Park (Eds.), Cognitive neuroscience of aging (pp. 107-131). New York: Oxford University Press.

Glabus, M. F., Horwitz, B., Holt, J. L., Kohn, P. D., Gerton, B. K., Callicott, J. H., et al. (2003). Interindividual differences in functional interactions among prefrontal, parietal and parahippocampal regions during working memory. Cerebral Cortex, 13, 1352-1361.

Grady, C. L., McIntosh, A. R., Bookstein, F., Horwitz, B., Rapoport, S. I., \& Haxby, J. V. (1998). Age-related changes in regional cerebral blood flow during working memory for faces. Neuroimage, 8, 409-425.

Gunning-Dixon, F. M., \& Raz, N. (2000). The cognitive correlates of white matter abnormalities in normal aging: A quantitative review. Neuropsychology, 14, 224-232.

Honey, G. D., Fu, C. H., Kim, J., Brammer, M. J., Croudace, T. J., Suckling, J., et al. (2002). Effects of verbal working memory load on corticocortical connectivity modeled by path analysis of functional magnetic resonance imaging data. Neuroimage, 17, 573-582.

Ito, H. T., \& Schuman, E. M. (2008). Frequency-dependent signal transmission and modulation by neuromodulators. Frontiers in Neuroscience, 2, 138-144.

Jenkinson, M., Bannister, P., Brady, M., \& Smith, S. (2002). Improved optimization for the robust and accurate linear registration and motion correction of brain images. Neuroimage, 17, 825-841.

Jenkinson, M., \& Smith, S. (2001). A global optimisation method for robust affine registration of brain images. Medical Image Analysis, 5, 143-156.

Jonides, J., Lewis, R. L., Nee, D. E., Lustig, C. A., Berman, M. G., \& Moore, K. S. (2008). The mind and brain of short-term memory. Annual Review of Psychology, 59, $193-224$

Jonides, J., Schumacher, E. H., Smith, E. E., Lauber, E. J., Awh, E., Minoshima, S., et al. (1997). Verbal working memory load affects regional brain activation as measured by PET. Journal of Cognitive Neuroscience, 9, 462-475.

Jonides, J., Smith, E. E., Koeppe, R. A., Awh, E., Minoshima, S., \& Mintun, M. A. (1993). Spatial working memory in humans as revealed by PET. Nature, 363, 623-625.

Kaasinen, V., Vilkman, H., Hietala, J., Nagren, K., Helenius, H., Olsson, H., et al. (2000). Age-related dopamine D2/D3 receptor loss in extrastriatal regions of the human brain. Neurobiology of Aging, 21, 683-688.

Kirchhoff, B. A., \& Buckner, R. L. (2006). Functional-anatomic correlates of individual differences in memory. Neuron, 51, 263-274.

Klingberg, T., O’Sullivan, B. T., \& Roland, P. E. (1997). Bilateral activation of fronto-parietal networks by incrementing demand in a working memory task. Cerebral Cortex, 7 , 465-471.

Kondo, H., Morishita, M., Osaka, N., Osaka, M., Fukuyama, H., \& Shibasaki, H. (2004). Functional roles of the cingulo-frontal network in performance on working memory. Neuroimage, 21, 2-14.

Kondo, H., Osaka, N., \& Osaka, M. (2004). Cooperation of the anterior cingulate cortex and dorsolateral prefrontal cortex for attention shifting. Neuroimage, 23, 670-679. 
Kray, J., \& Lindenberger, U. (2000). Adult age differences in task switching. Psychology and Aging, 15, 126-147.

Kriegeskorte, N., Simmons, W. K., Bellgowan, P. S., \& Baker, C. I. (2009). Circular analysis in systems neuroscience: The dangers of double dipping. Nature Neuroscience, $12,535-540$.

Li, S.-C., \& Sikström, S. (2002). Integrative neurocomputational perspectives on cognitive aging, neuromodulation, and representation. Neuroscience \& Biobehavioral Reviews, 26, 795-808.

Lindenberger, U., \& Baltes, P. B. (1997). Intellectual functioning in old and very old age: Cross-sectional results from the Berlin Aging Study. Psychology and Aging, 12, 410-432.

Lindenberger, U., \& Ghisletta, P. (2009). Cognitive and sensory declines in old age: Gauging the evidence for a common cause. Psychology and Aging, 24, 1-16.

Lindenberger, U., Mayr, U., \& Kliegl, R. (1993). Speed and intelligence in old-age. Psychology and Aging, 8, $207-220$

Lindenberger, U., Scherer, H., \& Baltes, P. B. (2001). The strong connection between sensory and cognitive performance in old age: Not due to sensory acuity reductions operating during cognitive assessment. Psychology and Aging, 16, 196-205.

Madden, D. J., Bennett, I. J., \& Song, A. W. (2009). Cerebral white matter integrity and cognitive aging: Contributions from diffusion tensor imaging. Neuropsychology Review, 19, 415-435.

Manoach, D. S., Schlaug, G., Siewert, B., Darby, D. G., Bly, B. M., Benfield, A., et al. (1997). Prefrontal cortex fMRI signal changes are correlated with working memory load. NeuroReport, 8, 545-549.

Mattay, V. S., Fera, F., Tessitore, A., Hariri, A. R., Berman, K. F., Das, S., et al. (2006). Neurophysiological correlates of age-related changes in working memory capacity. Neuroscience Letters, 392, 32-37.

Mayr, U., \& Kliegl, R. (1993). Sequential and coordinative complexity: Age-based processing limitations in figural transformations. Journal of Experimental Psychology: Learning, Memory, \& Cognition, 19, 1297-1320.

Mesulam, M. M. (1990). Large-scale neurocognitive networks and distributed processing for attention, language, and memory. Annals of Neurology, 28, 597-613.

Meyer-Lindenberg, A., Nichols, T., Callicott, J. H., Ding, J., Kolachana, B., Buckholtz, J., et al. (2006). Impact of complex genetic variation in COMT on human brain function. Molecular Psychiatry, 11, 867-877.

Miller, E. K., \& Cohen, J. D. (2001). An integrative theory of prefrontal cortex function. Annual Review of Neuroscience, 24, 167-202.

Miyake, A. (2001). Individual differences in working memory: Introduction to the special section. Journal of Experimental Psychology: General, 130, 163-168.

Nagano-Saito, A., Leyton, M., Monchi, O., Goldberg, Y. K., He, Y., \& Dagher, A. (2008). Dopamine depletion impairs frontostriatal functional connectivity during a set-shifting task. Journal of Neuroscience, 28, 3697-3706.

Nagel, I. E., Preuschhof, C., Li, S.-C., Nyberg, L., Bäckman, L., Lindenberger, L., et al. (2009). Performance level modulates adult age differences in brain activation during a spatial working memory task. Proceedings of the National Academy of Sciences, U.S.A., 106, 22552-22557.

Narayanan, N. S., Prabhakaran, V., Bunge, S. A., Christoff, K., Fine, E. M., \& Gabrieli, J. D. (2005). The role of the prefrontal cortex in the maintenance of verbal working memory: An event-related fMRI analysis. Neuropsychology, 19, 223-232.
Nyberg, L., Dahlin, E., Stigsdotter Neely, A., \& Bäckman, L. (2008). Neural correlates of variable working memory load across adult age and skill: Dissociative patterns within the fronto-parietal network. Scandinavian Journal of Psychology, 50, 41-46.

O'Sullivan, M., Jones, D. K., Summers, P. E., Morris, R. G. Williams, S. C., \& Markus, H. S. (2001). Evidence for cortical "disconnection" as a mechanism of age-related cognitive decline. Neurology, 57, 632-638.

Owen, A. M., McMillan, K. M., Laird, A. R., \& Bullmore, E. (2005). $N$-back working memory paradigm: A meta-analysis of normative functional neuroimaging studies. Human Brain Mapping, 25, 46-59.

Park, D. C., Lautenschlager, G., Hedden, T., Davidson, N. S., Smith, A. D., \& Smith, P. K. (2002). Models of visuospatial and verbal memory across the adult life span. Psychology and Aging, 17, 299-320.

Park, D., \& Reuter-Lorenz, P. (2009). The adaptive brain: Aging and neurocognitive scaffolding. Annual Review of Psychology, 60, 1-24.

Petrides, M., \& Pandya, D. N. (1984). Projections to the frontal cortex from the posterior parietal region in the rhesus monkey. Journal of Comparative Neurology, 228 $105-116$

Pfefferbaum, A., Sullivan, E. V., Hedehus, M., Lim, K. O., Adalsteinsson, E., \& Moseley, M. (2000). Age-related decline in brain white matter anisotropy measured with spatially corrected echo-planar diffusion tensor imaging. Magnetic Resonance in Medicine, 44, 259-268.

Postle, B. R. (2006). Working memory as an emergent property of the mind and brain. Neuroscience, 139, 23-38.

Raz, N. (2005). The aging brain observed in vivo. Differential changes and their modifiers. In R. Cabeza, L. Nyberg, \& D. C. Park (Eds.), Cognitive neuroscience of aging: Linking cognitive and cerebral aging (pp. 19-57). New York: Oxford University Press.

Raz, N., Lindenberger, U., Rodrigue, K. M., Kennedy, K. M., Head, D., Williamson, A., et al. (2005). Regional brain changes in aging healthy adults: General trends, individual differences and modifiers. Cerebral Cortex, 15, 1676-1689.

Reuter-Lorenz, P. A. (2002). New visions of the aging mind and brain. Trends in Cognitive Sciences, 6, 394-400.

Reuter-Lorenz, P. A., \& Cappell, K. (2008). Neurocognitive aging and the compensation hypothesis. Current Directions in Psychological Science, 18, 177-182.

Reuter-Lorenz, P. A., \& Lustig, C. (2005). Brain aging: Reorganizing discoveries about the aging mind. Current Opinion in Neurobiology, 15, 245-251.

Rogers, B. P., Morgan, V. L., Newton, A. T., \& Gore, J. C. (2007). Assessing functional connectivity in the human brain by fMRI. Magnetic Resonance Imaging, 23, 1347-1357.

Rypma, B., \& D'Esposito, M. (1999). The roles of prefrontal brain regions in components of working memory: Effects of memory load and individual differences. Proceedings of the National Academy of Sciences, 96, 6558-6563.

Rypma, B., Prabhakaran, V., Desmond, J. E., Glover, G. H., \& Gabrieli, J. D. (1999). Load-dependent roles of frontal brain regions in the maintenance of working memory. Neuroimage, 9, 216-226.

Sambataro, F., Murty, V. P., Callicott, J. H., Tan, H. Y., Das, S., Weinberger, D. R., et al. (2008). Age-related alterations in default mode network: Impact on working memory performance. Neurobiology of Aging, 31, 839-852.

Schmiedek, F., Lövdén, M., \& Lindenberger, U. (2009). On the relation of mean reaction time and intraindividual reaction time variability. Psychology and Aging, 24, 841-857. 
Schneider-Garces, N. J., Gordon, B. A., Brumback-Peltz, C. R., Shin, E., Lee, Y., Sutton, B. P., et al. (2010). Span, CRUNCH and beyond: Working memory capacity and the aging brain. Journal of Cognitive Neuroscience, 22, 655-669.

Servan-Schreiber, D., Printz, H., \& Cohen, J. D. (1990). A network model of catecholamine effects: Gain, signal-to-noise ratio, and behavior. Science, 249, 892-894.

Smith, S. M., Jenkinson, M., Woolrich, M. W., Beckmann, C. F., Behrens, T. E., Johansen-Berg, H., et al. (2004). Advances in functional and structural MR image analysis and implementation as FSL. Neuroimage, 23(Suppl. 1), S208-S219.
Suhara, T., Fukuda, H., Inoue, O., Itoh, T., Suzuki, K., Yamasaki, T., et al. (1991). Age-related changes in human D1 dopamine receptors measured by positron emission tomography. Psychopharmacology, 103, 41-45.

Todd, J. J., \& Marois, R. (2005). Posterior parietal cortex activity predicts individual differences in visual short-term memory capacity. Cognitive, Affective \& Behavioral Neuroscience, 5, 144-155.

Wechsler, D. (1981). The psychometric tradition-Developing the Wechsler Adult Intelligence Scale. Contemporary Educational Psychology, 6, 82-85.

West, R. L. (1996). An application of prefrontal cortex function theory to cognitive aging. Psychological Bulletin, 120, 272-292. 